\title{
Virtual Furniture Design Bureau: Distributed Design in Multi-Agent Environment Using Cloud Technologies
}

\author{
Starikov A.V.* \\ Voronezh State University of Forestry and Technologies \\ named after G.F. Morozov \\ Voronezh, Russia \\ e-mail: star123@yandex.ru
}

Starikova A.A.

Voronezh State University

Voronezh, Russia

e-mail: astar.srv@mail.ru

\author{
Meshkov D.A. \\ JSC "Concern "Sozvezdie" \\ Voronezh, Russia \\ e-mail:dameshkov@gmail.com
}

\author{
Bunakov P.Yu. \\ State Social and Humanitarian University \\ Kolomna, Russia \\ e-mail: pavel_jb@mail.ru
}

Lopatin A.K.

State Social and Humanitarian University

Kolomna, Russia

e-mail: ak_lopatin@mail.ru

\author{
Pletnev A.A. \\ LLC "Bazis-Center" \\ Kolomna, Russia \\ e-mail: zix@bazissoft.ru
}

\begin{abstract}
One of the most important tasks of creating a digital furniture company is to ensure the conditions for the implementation of design and technological preparation of production (DTPP) using effective methods and automation tools. At present, custom-made industrial production of cabinet furniture has become quite widespread, focused on the needs of end users of furniture products and imposing stringent requirements for the timing and quality of DTPP. However, only a few domestic furniture companies have the necessary financial and human resources for the rapid development of high-quality projects. The features of the approach to solving the problems of the DTPP, which uses the main provisions of the concept of the virtual furniture design bureau (VFDB), are considered. This concept involves the organization of a unified system environment for performing the distributed design of complex products of cabinet furniture and manufacturing processes for their orders. The design activity of VFDB consists in the development of object structural attribute models (OSAM), which are a digital representation of products and basic technological processes of their manufacture. Organizationally, the VFDB is a virtual structure whose stationary core is a design center interacting with design studios, which, in turn, interact with many freelancers (individual designers, design engineers, technologists). To simulate a managed design environment, it is proposed to use a multi-agent approach, involving the interaction of various types of agents involved in project work. When automating the solution of control problems in a multi-agent environment, an information model of the distributed design process is used in the form of a series-parallel design pipeline. The important task for the functioning of the VFDB is to provide each participant in the design process with software tools that allow developing new product designs and coordinating joint design activities efficiently. The access to software design tools can be implemented using the capabilities of cloud technologies.
\end{abstract}

The features of using time-based remote licensing of CAD software modules adapted for applying in cloud computing are considered.

Keywords - custom-made industrial furniture production, virtual furniture design bureau, multi-agent environment, distributed design, distributed design management system, cabinet furniture CAD system, cloud technologies.

\section{INTRODUCTION}

The urgency of creation and efficient use of virtual design bureaus in various industries is very important. This is due to the tightening of requirements for the implementation of projects (for example, reducing the design time, improving the quality of projects and others), as well as the limited capabilities of design organizations to implement them for various reasons: increasing the complexity of the designed facilities, an acute shortage of professional staff, insufficient financial resources for modernization of projects infrastructure and others.

A similar situation has discovered in the domestic furniture industry. Most of Russian furniture enterprises are small and medium-size businesses, i.e. they do not have sufficient financial and well-trained human resources. It does not allow them to provide a design and technological preparation of high-quality production solutions. Many domestic furniture enterprises implement custom-made industrial production of cabinet furniture, which makes very stringent requirements for the quality and timing of the design of furniture products. At the same time, only an insignificant part of furniture manufacturers (mainly large and a number of medium-sized enterprises) have their own design bureaus (departments) that 
ensure the rapid expansion and updating of the range of products.

Therefore, it is advisable to develop the concept of a virtual furniture design bureau (VFDB), which proposes the organization of a system environment for performing the processes of distributed design of products and ensembles of cabinet furniture for orders of various manufacturers (mainly small and medium-sized enterprises). In this case, "virtuality" means that the participants in the design processes are spaced spatially (geographically) and temporally.

It should be noted that one of the most important tasks for the functioning of the VFDB is the implementation of a unified managed design work environment that provides each of the participants in the design process with tools to efficiently develop projects, as well as coordinate their joint actions and manage the process.

Organizationally, the VFDB includes a design center (central office) and design studios that interact with many individually working designers, design engineer and technologists, i.e. actually freelancers. The objectives of the design center are: receiving orders for the development of products from furniture manufacturers, maintaining operational communication with them, distributing received orders between design studios, which, in turn, interact with freelancers, coordinating the collaboration of design studios, quality control of completed projects, delivery of completed projects to customers.

The VFDB methodological setting is focused on the implementation of design tasks for the preparation of model ranges of furniture products (especially with regard to the design of cabinet furniture), i.e. the main result of the work in this case is the preparation of model libraries and the corresponding design and technological documents presented in digital form.

Managed distributed design environment involves the full using of the capabilities provided by the Internet, including the physical environment of data transfer, network data exchange protocols, various services, including cloud computing. Specialized tools used by VFDB can be represented by software tools for product engineering, management of distributed project development, interaction with customers, i.e. means of integration of information spaces of furniture enterprises and VFDB.

It should be noted that there has been the increased interest of CAD developers in information technologies that provide GRID and cloud computing, both abroad and in our country. In particular, well-known domestic systems, such as COMPAS-3D (CJSC “ASCON”), T-FlexCAD (CJSC “TopSystems") and some others, have been tested for compatibility with NVIDIAGRID technology. This technology allows organizing virtual workplaces for design work as an alternative to using high-performance workstations.

In addition, a number of domestic CAD developers (for example, CJSC “ASCON”, LLC "BAZIS-Center" and others) are trying to provide their services using cloud technologies. Thus, the construction of a design work environment within the framework of the VFDB involves the using of modern information technologies that provide opportunities for the distributed project development.

\section{THEORETICAL AND METHODOLOGICAL BACKGROUND}

The main limitation of the existing CAD software for furniture is an exclusive orientation to the furniture products geometric models using that require entering a lot of "executive" coordinates, which contributes to the subjective errors introduction. In addition, the traditional geometric model complicates the distributed (combined) design, because it does not provide the amount of information sufficient for its organization.

To overcome the above limitations within the framework of the developed concept of cabinet furniture error-free design and production (EFDP), a high-level structural models complex is used, where are represented by the object structural-attributive model (OSAM) [1].

OSAM provides a multidimensional representation of the product design, which can be described by the following five components:

$$
M_{\text {OSAM }}=<G, S, R, K, T>_{\alpha},
$$

where $G$ - geometric, $S$ - structurally-attributive, $R$ calculation-analytical, $K$ - constructive, $T$ - the production and technological aspects of the project, $\alpha-$ the level of abstraction of the model.

In the concept of EFDP, the following levels of abstraction are defined, conditionally called fundamental, constructive, working. At the level of fundamental abstraction corresponding to the initial stage of the engineering stage, the tasks of structural and parametric synthesis of the product are performed. At this level, a number of fundamental parameters of the prototype model are determined, representing a certain class (set) of furniture products.

At the level of constructive abstraction corresponding to the engineering stage, it is possible to include generalized information about production processes in the prototype model of the designed product. This information is presented by a set of design and technological requirements and limitations (DTRL), as well as appropriate methods for their control.

At the level of working abstraction, which corresponds to the stage of reengineering, the formal parameters presented in the prototype model are replaced with their specific values obtained during the acceptance of the order when interacting with the customer. In other words, at this level, an essential transformation of the model takes place. It ceases to be the prototype and provides complete information about a particular product of cabinet furniture.

Thus, the separation of the OSAM presentation by the levels of abstraction in the design of cabinet furniture allows:

- organizing relatively independent design processes, distributed in time and space, at each of the indicated levels of abstraction;

- ensuring the work of various specialists (designers, designer engineers, technologists) in accordance with 
their competence and authority, thereby reducing the impact of the subjective factor on the quality of project preparation.

OSAM can also be considered as a combination of the following structural models:

$$
M_{\text {OSAM }}=M_{\text {OSAM }}^{S S} \cup M_{\text {OSAM }}^{\text {StAt }} \cup M_{\text {OSAM }}^{\text {GrAn }} \cup M_{\text {OSAM }}^{\text {Geom }} \cup M_{\text {OSAM }}^{\text {DTRL }},
$$

where $M_{O S A M}^{S S}$ - sketch-structural model, $M_{O S A M}^{S t A t}$ - structuralattributive model, $M_{O S A M}^{G r A n}$ - graph-analytical model, $M_{O S A M}^{G e o m}-$ geometric model, $M_{O S A M}^{D T R}-$ DTRL complex. The aspectual representations $S, G, R, K$, and $T$ presented above are implemented by structural models. At the same time, aspectual representations, as a rule, turn out to be wider, since they include information used by more than one model.

The sketch-structural model $M_{O S A M}^{S S}$ uses the structural classification of the elements of the product and provides a preliminary (enlarged) coordinate representation of the furniture structure. Identification of elements is carried out using a system of related concepts (levels), with which the design of any product of cabinet furniture can be represented in the form of a planar graph: $G_{p}(V, P)$. Each vertex $(V)$ of the graph refers to one of four levels: a product, section, block or part; edges $(R)$ connect the corresponding vertices of different levels

The structural-attributive model $M_{O S A M}^{S t A t}$ can be represented by the following union:

$$
M_{O S A M}^{S t A t}=\left(\bigcup_{i=1}^{N} s_{i}\right) \cup\left(\bigcup_{i=1}^{M} \bigcup_{\substack{k=1 \\ L_{j}}}^{M} \bigcup_{\substack{j=1, j \neq i}}^{M} S_{i, k}^{j}\right)
$$

where $N$ - the number of geometric elements of a furniture product; $M$ - the number of levels of hierarchical decomposition of the design object; $L_{j}$ - power of the set of structural elements of the $j$-th level of of decomposition; $s_{i}$ vector of structural parameters of the $i$-th element, consisting of functional, structural and technological parameters, respectively; $S_{i, k}^{j}$ - the relationship vector of the $k$-th structural element of the $i$-th level of decomposition witch elements of other levels.

The graph-analytical model $M_{O S A M}^{G r A n}$ uses a special notation, which combines graphic and linguistic tools and is used to describe the structure of the design object. This notation can be considered as the following multi-set:

$$
M_{O S A M}^{G r A n}=\left\{S_{G r A n}, R_{G r A n}\right\},
$$

where $S_{G r A n}$ - a set of furniture in accordance with the information presented of a planar graph $G_{P}(V, R) ; R_{G r A n}-$ set of operators describing relations between elements.

The geometric model $M_{O S A M}^{\text {Geom }}$ can be represented by the following combination:

$$
M_{\text {OSAM }}^{\text {Geom }}=\left(\bigcup_{i=1}^{N} g_{i}\right) \cup\left(\bigcup_{\substack { i=1 \\
\begin{subarray}{c}{j=1, j \neq i{ i = 1 \\
\begin{subarray} { c } { j = 1 , \\
j \neq i } }\end{subarray}}^{N} F_{i, j}^{1}\right) \cup\left(\bigcup_{i=1}^{N} \bigcup_{j=1}^{M} F_{i, j}^{2}\right) \cup\left(\bigcup_{k=1}^{P} f_{k}\right),
$$

where $N$ - the number of geometric elements in model of the design object; $M$ - the number of levels of hierarchical decomposition of the design object; $P$ - the number of external structurally conjugated relationships of the object in the model of furniture; $g_{i}$ - vector of geometrical parameters of the $i$-th element, including overall parameters, coordinates of the characteristic points of the parametric curves that define the shape of the parts and visualization parameters; $F_{i, j}{ }^{1}, F_{i, j}^{2}$ internal conjugation of elements belonging to different hierarchical levels of the object and different elements of the same hierarchical level, respectively; $f_{k}$ - external interfaces of the objects in the furniture model.

The DPRL complex can be represented by the following union:

$$
M_{\text {OSAM }}^{\text {DPRL }}=\left(\bigcup_{i=1}^{N} \bigcup_{j=1}^{M} \bigcup_{k=1}^{P} K_{i, j, k}\right),
$$

where $N$ - the number of geometric elements of the design object; $M$ - the number of levels of hierarchical decomposition of the design object; $P$ - the number of external structurally conjugated relationships of the object in the model of furniture product; $K_{i, j, k}$-design and technological requirements for the design object at the $j$-th level of decomposition of the current level of abstraction.

In the model $M_{\text {OSAM }}$, the DPRL complex, which represents the constructive and industrial-technological aspects, is implemented by a formal relationships and algorithms that determine the admissibility of the formed design decisions.

The multidimensional presentation of the project described above allows implementing the cabinet furniture combined design at various levels of abstraction. For this, the process of designing furniture products is represented by two separate stages, called the prototype model engineering and reengineering. At the engineering stage, carried out by the designer-technologist, a prototype product model is formed, in which the requirements of the technical task, as well as data on the technological processes of the enterprise, must be taken into account.

In addition, restructuring algorithms that are designed to determine the rules for compiling prototype models into models of specific furniture products, as well as control algorithms that guarantee the accuracy of design operations performed at the reengineering stage, should be included in the prototype model.

At the stage of prototype model reengineering, performed by the designer upon receiving an individual order, two main design procedures are used:

- prototype model restructuring;

- prototype model compilation. 
The restructuring procedure provides the transformation of the prototype model without lowering the abstract representation level. Using reengineering tools, the furniture designer gets access to the fields of data structures that describe the prototype product model. At the same time, the correctness of data input into the fields is controlled by the restructuring algorithms that were set at the engineering stage, which substantially guarantees the error-free operations when working with the model.

The compilation procedure is carried out after the prototype model restructuring and ensures its conversion into a model of a specific furniture product, which contains the necessary information for the manufacture of the product on the production basis of the enterprise.

In view of the foregoing, it should be noted that the transition to custom-made industrial production of cabinet furniture entails qualitative changes in the design process, expressed in the using of a complex of high-level models that reduce the number of subjective errors and organize distributed design. The data structures and methods presented in OSAM can be used to initiate work that follows the design and development of the project and includes technological preparation of production, its material and technical support, warehouse and transportation services for the enterprise.

\section{DESIGN AND MANAGEMENT IN MULTIAGENT ENVIRONMENT OF VFDB}

The main tasks of managing technical projects are:

- the formation of the project structure;

- planning of the project processing process and its operational re-planning in accordance with the received design decisions;

- ensuring interaction and coordination of actions of various functional subsystems;

- ensuring and monitoring the logical integrity of the project; project status monitoring;

- accounting for changes made to the project;

- monitoring of the work schedule implementation;

- control and ensuring the reliability of information during design decisions storage and transfer of between functional subsystems and others.

These project management tasks importance increases significantly with the parallel execution of project procedures in a distributed data processing environment. In this case, the management tasks total complexity of becomes comparable with project tasks complexity, and their solution requires the development of special management methods and automated support tools [2].

To solve the automated control problems of a multi-agent medium of VFDB, it is necessary to choose a conceptual model of the distributed design process. As such a model, it is proposed to use a series-parallel design pipeline, which can be represented by the following metagraph:

$$
M G=\left(V, V_{m}, e, M_{e}\right),
$$

where $V$ - the set of vertices associated with agents; $V_{m}$ - the set of meta-vertices uniting the sets of agents $V$ and the design procedures executed by them; $e$ - the set of arcs defined on the set $V ; M_{e}$ - the set of meta-arcs defined on the set $V_{m}$.

Thus, in the model represented by the MG metagraph, the vertices are the agents and the design procedures performed by him, combined into meta-vertices, the links between which form meta-arcs. Many of the meta-vertices and meta-arcs of the MG metagraph represent the VFDB multi-agent structure. At the same time, every change in this structure allows tracking all affected relationships between agents.

To build a multi-agent environment, the project activities analysis is carried out within the VFDB framework, an agents types' classification is presented, and their functions are determined. Let us consider as an example the following classification of agents:

- $\quad$ agent-customer (AC);

- agent-coordinator (AK);

- agent-executor (AE);

- agent-subordinator (AS).

The main functions list defined for each type of agent is presented in the table below.

TABLE I. THE MAIN FUNCTIONS OF THE AGENTS OF VFDB

\begin{tabular}{|c|c|}
\hline Agent type & The main functions that the agent performs \\
\hline $\mathbf{A C}$ & $\begin{array}{l}\text { - Formation and dispatch of the order. } \\
\text { - Evaluation and acceptance of work performed. }\end{array}$ \\
\hline AK & $\begin{array}{l}\text { - Processing requests from AC (formation of a pool of orders). } \\
\text { - Comparison of multiple orders with multiple active AE and } \\
\text { distribution of the order flow. } \\
\text { - Formation of a request for initiation of reengineering of } \\
\text { existing OSAM products. } \\
\text { - Formation of a request to create an OSAM product. } \\
\text { - Coordination of the outline design of the product with AC. }\end{array}$ \\
\hline $\mathbf{A E}$ & $\begin{array}{l}\text { - Reengineering OSAM products. } \\
\text { - Development of technical specifications for the designed } \\
\text { product. } \\
\text { - Performing OSAM product engineering. } \\
\text { - Formation of OSAM products database. } \\
\text { - Development of electronic submission of design and } \\
\text { technological documentation (DTD). } \\
\text { - Correction of electronic submission of DTD (if necessary). }\end{array}$ \\
\hline $\mathbf{A S}$ & $\begin{array}{l}\text { - Tracking the implementation of the project (order). } \\
\text { - Evaluation and optimization of load distribution between AE. } \\
\text { - Operational management of the interaction of AC, AK, AE. } \\
\text { - Assignment of authority and distribution of duties of agents. } \\
\text { - Control (monitoring) of the design process. } \\
\text { - Analysis of the progress of work on projects. } \\
\text { - Prompt response to exceptional situations in the course of } \\
\text { design work. }\end{array}$ \\
\hline
\end{tabular}

It should be noted that when implementing the multi-agent medium of VFDB, it is supposed to clarify the agent types and their functions classification. For each type of agent, a basic model of behavior should be provided, supplemented by possible scenarios sets of work in a distributed design environment. 
A generalized design operation performed to implement the $\mathrm{AE}$ request is reducing the OSAM abstraction level, i.e. its translation from the sketch coordinates space to the product executive coordinates space. This operation content is considered in detail below in the theoretical and methodological premises view discussed above.

The product model construction at the engineering stage is developed at the fundamental and constructive abstraction levels and provides for the combination of a parametric geometric model of the product with object aspects represented by sketch-structural, structural-attributive and graph-analytical models, as well as a generalized set of DPRL.

The design subset of DPRL is determined by the geometric parameters of the individual elements and their relative position, as well as the functional purpose of the product, while the technological subset is determined by the correspondence of the design operations to the existing machine equipment and the manufacturing processes being implemented. This allows accumulating in the model a sufficient set of abstract parameters that determine the information support of individual project operations at a particular enterprise.

Thus, the process of implementing an order at VFDB represents a phased transition from abstract descriptions at the early stages of design to the formation of full software and documentary support for the technological process of manufacturing products using real equipment and using the necessary materials.

At the fundamental abstraction level, which provides a structural and parametric synthesis problems solution, the model is invariant to all enterprise's business processes aspects, being, in fact, a design pattern. It contains formal positions that can be further defined at the following abstraction levels. The model formation technology involves the definition of relationships between objects without analytical descriptions, by supplementing the logical relationships set with new relationships. Their type and character are automatically determined based on the associated objects properties and the order in which each relationship is included in the model.

At the constructive abstraction level, an uncertainties number due to the specifics of the project operations implementation for a particular enterprise are eliminated without changing the outline character of the model. This creates a structural-attributive description (the reengineering algorithms formalized description) and DPRL (information on the design and technological features of the production preparation). The final transition to the executive coordinates is made at the working abstraction level, at the output of which the model is presented in the applied CAD system format. Using the model in this format is a sufficient condition for the transition to the production technological preparation process.

It should be noted that the proposed object models using makes it possible to solve a number of urgent problems related to the efficient work of VFDB: organize interaction with any automation level enterprises, up to digital production, actively use distributed design technologies, minimize subjective errors in the design process, and reduce the time for order execution.

The distributed design and centralized management implementation in a multi-agent environment of VFDB provides for the provision of all agents with the necessary software and information support. For this purpose, we can apply the so-called cloud technology, using of which in relation to CAD system is discussed below.

\section{TIME-BASED REMOTE LICENSING TECHNOLOGY}

Distributed design technologies are the basis for the functioning of the VFDB, since they allow the centralized order management organization, provide optimal means of communication for agents, solve the centralized storage and processing of information problem, minimize software and hardware costs, eliminate intellectual property protection problems, increase agent mobility and achieve transparency in relation to the used software platforms.

The information flows scheme in the VFDB distributed structure shown in Figure 1.

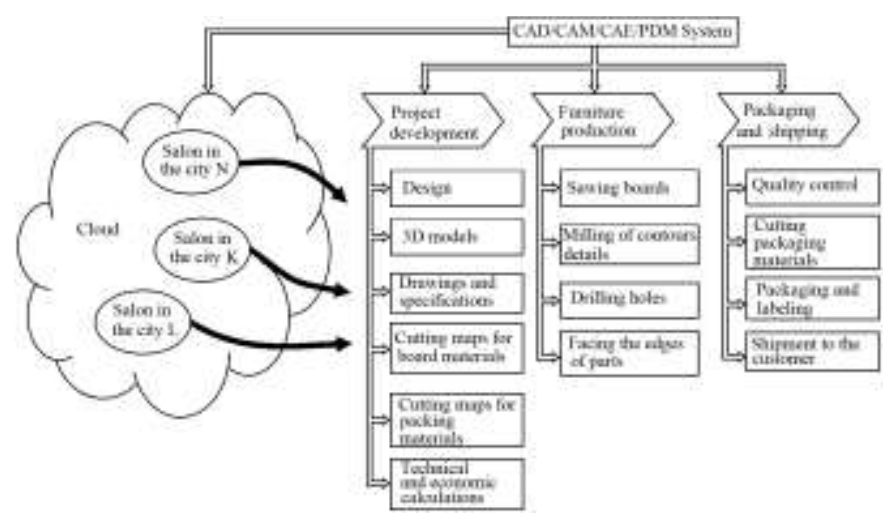

Fig. 1. VFDB information flows

The most well-known technologies for providing cloud services show low efficiency when applied in the computeraided design, because they do not solve the problem of quickly transporting three-dimensional large-volume content from a server to a host, which is typical for CAD. It is necessary to take into account that the transition to high-speed data channels and the software adapted to the 3D design requires financial costs increasing, comparable to the costs of organizing the corresponding local workstations [3]

Time-based Remote Licensing (TBL) is a hybrid approach combining cloud technologies with a local way of organizing CAD hardware. Its main task is to provide all VFDB agents with flexible, convenient and economically sound interaction mechanisms. TBL combines the principle of software rental and its local installation. It allows changing their quantitative ratio "transparently" for production in accordance with the current technological situation. This makes it possible to eliminate the need for physical transportation of protection tokens against unlicensed access to agents and use a single security key for which the necessary performance characteristics for all agents are specified. In addition, network 
traffic is minimized (an experiment showed that the $3 \mathrm{G}$ modem's performance is sufficient for the entire VFBD infrastructure to work), a high level software protection is provided, and a mechanism for its operational updating is implemented.

Structurally, the TBL is represented by a combination of two components:

$$
T B L=<W, L S>,
$$

where $W-$ a modular web shell embedded in the site of the software company; $L S$ - licensing system. The web shell provides agents with all the necessary tools for teamwork, including interfaces with e-commerce and Internet acquiring systems. Interaction security when working in it is provided by the SSL protocol (Secure Sockets Layer). The licensing system is used to protect software from illegal replication and control of limited indicators. Its distinguishing feature is the minimal identification traffic amount. This, in combination with the distributed licensing servers' allocation, allows providing high fault tolerance and stable operation of the VFDB even when low speed communication channels are used.

\section{ECONOMIC ASPECTS OF USING THE MULTIAGENT ENVIRONMENT OF VFDB}

The interaction of the coordinator agent with the customer agent within the environment of the VFDB suggests the need to solve a number of economic problems related to pricing. The accuracy and their decision's speed is a direct factor in ensuring the competitiveness of decisions.

The methodology for the technical and economic calculations implementation in the organization of the VFDB is based on the technological support concept. The graphical and analytical OSAM description at any level of abstraction includes information about materials and component parts, presented in outline or executive coordinates (vector of basic materials). This makes it possible to accurately calculate their number for all items.

The second groups of materials are not present obviously in the graph-analytical description, but can be specified as a concomitant - a quantitative functional dependence on a certain nonempty subset of the basic materials elements vector:

$$
K\left(m_{i}\right)=\varphi_{i}\left(M_{i}\right)
$$

where $K\left(m_{i}\right)$ - the amount of $i$-th related material; $\varphi_{i}-$ a function that determines the dependence of the number of units of measure of related material on the totality of units of elements of the $i$-th subset of elements of the vector of basic materials; $M_{i}-$ a subset of the main materials for which the amount of the $i$-th related material is calculated. The number of vectors of related materials that are associated with a subset of the base materials is not limited.

A similar relationship holds for related materials, as new chains of attendance generators. A natural limitation of the depth of the attendance is the absence of cyclic groups. The separation of materials into two subsets is dynamic and is implemented at the level of the agent-executor.

With any subset of materials, depending on the level of abstraction, a generalized or real technological operation can be associated with the following assumptions that do not reduce the generality of the proposed approach:

- the functional dependence of the complexity of the operation on the materials generating it appears to be a curried function;

- every technological operation can be associated with a subset of materials whose consumption is functionally dependent on the number of materials generating it; the source of every escort chain is a subset of the basic materials.

The mathematical expression for determining the complexity of processing the $i$-th subset of materials will be:

$$
L\left(M_{i}\right)=\sum_{k=1}^{n} \rho_{i k}\left(m_{i k}\right) ; m_{i k} \in M_{i},
$$

where $n-$ the power of the $\mathrm{i}$-th subset of materials; $\rho_{i k}-\mathrm{a}$ function that determines the dependence of the specific complexity of the implementation of the processing operation of the $k$-th material from the $i$-th subset.

Combining the above expressions, we can obtain an algorithm for the automatic calculation of the material consumption of a furniture product, the complexity of its manufacture and cost. This information is available for analysis and decision-making by an agent of the VFDB.

\section{PRACTICAL IMPLEMENTATION OF ELEMENTS OF A DISTRIBUTED DESIGN ENVIRONMENT IN CAD SYSTEM BAZIS}

The VFDB concept reflects the modern principles of creating a competitive furniture business. Its theoretical provisions were used in the development of cloud services for the integrated automation system of furniture enterprises BAZIS. At present, three agent synchronization services have been put into operation:

- an agent-customer and an agent-coordinator (order synchronization);

- an agent-coordinator and agent-subordinator (updating price lists);

- an agent-customer and an agent-executor (commercial cutting).

The AK operation scheme in the service of order synchronization includes the following actions: analysis of the functional, aesthetic and financial needs of the $\mathrm{AC}$, modeling of a possible variant of their implementation, coordination of the parameters of the future product with the AC, transmission of information from the AK to analyze the feasibility of decisions, prompt receipt of possible structural or technological adjustments re-approval with AC. The agreed version of the order receives the status "in production". Its passage through the manufacturing process chain is automatically reflected in the 
$\mathrm{AK}$, which allows providing $\mathrm{AC}$ with up-to-date information on the status of its order at any time.

The service for synchronizing orders supports a number of mechanisms to ensure the correctness of information and protect it from unauthorized actions: blocking order changes after it receives a certain status, ranked access to databases, creating and storing a complete history of working on an order with the ability to access any previous version, and an operational communication system with any of the agents.

The price list update service is initiated by the AS in those cases when the lineup or price policy of the base enterprise changes. For this, the AS prepares the necessary information array and uploads it to the cloud storage. The software installed in AK automatically monitors the fact of the new price lists appearance and the degree of their correlation with existing ones, after which it makes changes to local databases in the incremental background mode. If at this moment the AK is in dialogue mode with the $\mathrm{AC}$, special measures are automatically taken to exclude the possibility of an incorrect ordering.

In the case of a geographically distributed network, an AS has the ability to create group interfaces that reflect the different status of individual coordinating agents. This allows users to individualize the nature of the interaction between AS and AK with minimal cost, because a single price list is automatically adjusted before sending to each group of coordinating agents. Logging the update process allows AS to monitor the process and take the necessary measures in case of emergency situations.

The commercial cutting service is relevant for enterprises having a business such as "production as a service". In this case, a third-party furniture company that does not have the ability to implement certain technological operations can act as an AC. Currently, commercial cutting is implemented for cutting and cladding sheet materials, cutting molded materials, as well as shaping and milling operations. In fact, it plays the role of the visual interface of $\mathrm{AC}$ with specialized $\mathrm{AE}$ software.

The commercial cutting service is implemented as an embedded module frame for the $\mathrm{AE}$ site. The administrative part of the frame implements the tasks of setting order processing parameters, generating individual AC settings, monitoring their activity and order processing. AE has at its disposal effective tools for ranking priorities and filtering orders, their distribution in technological areas, accurate costing based on the concept of technological assistance, operational interaction with AC

The client part of the frame is designed to enter the AC data of your order: overall dimensions, names and quantities of parts, type of edge lining and corner processing, presence and type of grooves, whole pattern. Based on this, a preliminary specification is formed; estimated cost and deadline are calculated. If this information is accepted by AC, the order is received by $\mathrm{AE}$ for analysis and possible adjustment according to the current production situation. The order is fixed after final agreement with the AC.
The execution of the order provides the optional ability to print identification tags with barcodes in the encoding system of his choice. At any time, the AC can get accurate information about the status of the order, as well as the necessary set of supporting documents.

The analysis of the experience of using cloud services at a number of furniture enterprises [4] showed the novelty and practical importance of the methodology for building VFDB, as well as the direction of further research in this area.

\section{DISCUSSION OF THE RESULTS}

When creating a working environment for VFDB, it is proposed to use a multi-agent approach to solve the problems of distributed design of cabinet furniture. In this case entities of the environment are agents of different types, which are able to show their activity in accordance with the scenarios defined for each type. When realizing their behavior, each agent should be able to use the software available for VFDB through the using of cloud technologies. The methodology for building a working environment for distributed design of CAD software BAZIS has been tested, which provides a number of cloud services for agents to perform various types of tasks.

In a multi-agent system (MAS), as a subject-oriented model for managing distributed computing, tasks to be solved are distributed among agents according to certain rules. Moreover, the distribution of tasks means assigning each agent a role, the complexity of which is determined based on the capabilities of the agent $[5,6]$. Research and development of MAS refers to the field of artificial intelligence (AI). The following three types of MAS structural organization are distinguished:

- distributed AI;

- decentralized AI;

- artificial life.

With regard to the computer-aided design problems, structures of the first type are the particular interesting when the MAS is created to find the most effective specific problems solution for a while ensuring coordination of the several intelligent agent actions. The following organizational problems are solved

1) optimal decomposition of tasks into sub-tasks, taking into account the capabilities of agents and the selected criterion for assessing the quality of the solution obtained;

2) functional distribution of powers between agents;

3) the organization of data exchange and resource allocation in the process of solving the problem.

It should be noted that the problem decomposition process into sub-tasks and the solutions inversing composition process found in the structure of the distributed AI are occurs under the single center control. When developing MAS with the structure of the distributed AI, a top-down design is used taking into account the roles defined for agents and the results of dividing the problem into sub-tasks. 
Recently, the role of the multi-agent approach in the development of automated systems that implement the design of objects in various fields of engineering and technology has been growing. At the same time, the distributed processing control systems problems in multi-agent environments are being actively developed. To create MAS, a number of special software tools have been developed [7, 8].

\section{CONCLUSION}

Within the framework of the VFDB, a single working environment is being formed in which intelligent agents with various powers participate (for example, agent-subordinator are granted access to the design work as a whole, including the provision of resources, coordination of the actions of agentexecutors, monitoring of the design work execution, authorization responses to technological routes, design, adjustment of the planned events list). Agents can also be programs (program agents), in particular, a program that implements the distribution of messages about the occurrence of important events in the design process (for example, transferring a design solution developed by a design procedure to a project repository).

To automate the control problems solution in a multi-agent environment, the distributed design process information model is required. Conceptually, the distributed design process can be represented as a series-parallel design pipeline. This is due to the fact that the technological design route can contain both information-dependent design procedures that allow only sequential execution and information-independent, allowing parallel execution if the necessary resources are available.

To solve the problem, it is necessary to develop invariant software tools that ensure the creation (generation), maintenance of the current state and work with the information model of the distributed design process.

It is also important to analyze the existing manufacturing infrastructure of enterprises and their human resources and possibility of their structural transformations in accordance with the digital economy requirements in order to solve management problems in a multi-agent VFDB environment $[9,10]$.

\section{References}

[1] A.V. Starikov, A.A. Starikova, V.N. Kharin, "The multi-aspect modeling in paralleled designing of the custom furniture products", pp. 308-313, 2009 [The informatization of the processes of open systems based on CAD, ASNI, DBMS and artificial intelligence systems (INFOS-2009), V Int. Sci. and Techn. Conf.] Vologda

[2] D.A. Meshkov, A.V. Starikov, "A formal representation of the process of product design of case furniture in multi-agent medium", Forestry Engineer. J., vol. 3, no. 15, pp. 205-213, 2014.

[3] P.Yu. Bunakov, A.A. Pletnev, "Problems of integrating cloud technologies with CAD", Herald of computer and inform. Technol., no. 2, pp. 9-14, 2015

[4] R. Bagaev, "BAZIS company and its role in the development of a furniture company", CAD and graphics, vol. 12 , no. 278 , pp. $76-82$ 2019.

[5] I.M. Diaconescu, G. Wagner, "Modeling and Simulation of Web-ofThings Systems as Multi Agent Systems", p. 248, 28-30 September 2015 [Multiagent System Technologies, 13th German Conf. MATES 2015]. Cottbus, Germany, 2015.

[6] T. Altameem, M. Amoon, "An agent-based approach for dynamic adjustment of scheduled jobs in computational grids", J. Comput. Syst. Sci. Intern., vol. 49, no. 5, pp. 765-772, 2010.

[7] I. Bychkov, G. Oparin, A. Tchernykh, A. Feoktistov, V. Bogdanova, S. Gorsky, "Conceptual Model of Problem-Oriented Heterogeneous Distributed Computing Environment with Multi-Agent Management", Proc. Computer Sci., vol. 103, pp. 162-167, 2017.

[8] S.V. Batishchev, P.O. Skobelev, "The main stage of the development of multiagent systems in a tool environment for creating Internet applications", Bull. of the Samara Sci. Center of the Russ. Acad. of Sci., vol. 4, no. 1, pp. 96-104, 2002.

[9] N.A. Serebryakova, I.V. Avdeev, "The content of structural transformations of the region's economy, adequate to the requirements of digitalization", Proc. of the Voronezh State Univer. of Engineer. Technol., vol. 80, no. 4, pp. 408-412, 2018. Retrieved from: https://doi.org/10.20914/2310-1202-2018-4-408-412

[10] N.A. Serebryakova, A.V. Petrikov, "The principles of design and the organization of functioning of innovative infrastructures in the conditions of the Industry 4.0", Proc. of the Voronezh State Univer. of Engineer. Technol., vol. 80, no. 4, pp. 384-387, 2018. Retrieved from: https://doi.org/10.20914/2310-1202-2018-4-384-387 